\title{
Masculinities and the Contemporary Irish Theatre
}

\author{
Martine Pelletier
}

revues.org

Édition électronique

URL : http://

etudesirlandaises.revues.org/2531

ISSN : 2259-8863
Éditeur

Presses universitaires de Rennes

\section{Édition imprimée}

Date de publication : 30 décembre 2011

Pagination : 198-199

ISSN : 0183-973X

Référence électronique

Martine Pelletier, "Masculinities and the Contemporary Irish Theatre ", Études irlandaises [En ligne], 36-2 | 2011, mis en ligne le 30 septembre 2011, consulté le 07 octobre 2016. URL : http:// etudesirlandaises.revues.org/2531

Ce document a été généré automatiquement le 7 octobre 2016.

(C) Presses universitaires de Rennes 


\title{
Masculinities and the Contemporary Irish Theatre
}

\author{
Martine Pelletier
}

\section{RÉFÉRENCE}

Brian Singleton, Masculinities and the Contemporary Irish Theatre, Basingstoke, Palgrave Macmillan, 2011, VIII + 228 p., ISBN 978-0-230-22280-9

1 Cet ouvrage de Brian Singleton est une intervention bienvenue dans le monde de la critique théâtrale irlandaise. Singleton s'intéresse depuis longtemps à des formes théâtrales peu traditionnelles et très internationales, privilégiant la recherche, l'innovation et la performance, la pratique de la scène plus que le texte. Il explique en introduction la genèse de l'ouvrage par la publication ces dernières années d'études inspirées par la théorie du genre qui ont permis aux voix féminines de se faire entendre, sur scène et dans le discours critique et la nécessité de sortir d'une conception binaire entre hégémonie masculine et effacement du féminin : "It was not another book about Irish men that was needed, but one that asked new questions. What kind of men and their representations precisely have been canonized? And what have been the challenges to those hegemonic representations at the latter end of the twentieth century, and in the twenty-first? To deconstruct both the representations and interventions of masculinities on the Irish stage, and to expose how particular masculinities also succumb to the oppressive drives of hegemonic forms of masculinity performed as patriarchy. » Hégémonie masculine et masculinité hégémonique sont interrogées, analysées, pièces contemporaines et mises en scènes récentes d'œuvres plus classiques à l'appui. La sélection des pièces est généreuse, souvent fort pertinente et parfois un rien aléatoire. On note un parti pris délibéré très compréhensible pour les jeunes dramaturges et une part congrue réservée aux dramaturges plus canoniques. En fait, l'ouvrage déborde du cadre strictement théâtral, ce qui est une de ses forces. Si Brian Singleton parvient effectivement à suivre les évolutions sur les scènes irlandaises des 
formes plurielles de masculinité il fait bien plus que cela : par le biais d'introductions aux différents chapitres il fait dialoguer éléments de contexte et œuvres théâtrales. Ainsi "Contesting Canons » aborde les formes de masculinité chez Synge et O'Casey au travers de la mise en scène controversés de The Plough and the Stars par Gary Hynes à l'Abbey Theatre en 1991 et de la réécriture/transposition du Playboy of the Western World par Roddy Doyle et Bisi Adigun, faisant de Christy un immigrant nigérian en 2007. Mais ce chapitre parle aussi de la critique théâtrale en Irlande et de la façon dont Gary Hynes a vu son mandat de directrice artistique de l'Abbey mis en cause. "Performing Patriarchy " s'attache aux figures des patriarches d'hier et d'aujourd'hui. C'est dans ce chapitre que Marina Carr, Sebastian Barry, Enda Walsh sont analysés judicieusement. «Monologies and Masculinies» étudie le lien spécifique entre masculinités plurielles et forme monologique, soulignant le paradoxe d'une représentation de l'abjection du masculin qui dépend d'une exhibition triomphante du pouvoir de l'acteur masculin en pleine maitrise de son art et de son corps. Le chapitre se clôt sur une réflexion aussi pertinente qu'inattendue: Brian Singleton médite sur le journal tenu par Ben Barnes pendant ses années aux commandes de l'Abbey entre 2000 et 2005 et récemment publié, arguant qu'il met en scène "a monologic negotiation of competing masculinities ». Avec "Quare Fellows " Singleton use des concepts diffusés par les «queer studies » pour déconstruire les binarités féminin/masculin et les représentations de l'homosexualité. C'est sans doute dans ce chapitre pivot de l'ouvrage que Singleton accorde au contexte, au hors scène, la place la plus grande. "Male Races» ajoute la dimension raciale à ces constructions/ déconstructions de masculinités plurielles et l'ouvrage se clôt sur les "Protestant Boys » de Gary Mitchell et autres dramaturges d'Irlande du Nord. Bien écrit, avec peu de jargon mais un solide cadre critique fourni par les études de genre, Masculinities and the Contemporary Irish Theatre offre au lecteur un cadre interprétatif pertinent et des microanalyses de grande qualité.

\section{AUTEURS}

\section{MARTINE PELLETIER}

Université François Rabelais-Tours 